\title{
An exceptional response to immunotherapy with chemotherapy in metastatic lung cancer
}

\author{
Xenia Elena BACINSCHI, Laurentia Nicoleta GALES, \\ Anca Florina ZGURA, Rodica Maricela ANGHEL \\ "Carol Davila" University of Medicine and Pharmacy, Bucharest, Romania
}

\begin{abstract}
Lung cancer is the leading cause of cancer death in men in Romania and more recently is a common cause of mortality among women. The main risk factor is smoking.

The prognosis of patients diagnosed with advanced lung cancer is generally considered poor, with a median survival of 8-10 months and a 2-year survival of no more than 10\%-20\%. The use of checkpoint immunity inhibitors in many malignancies, including lung cancer, has revolutionized the field of oncology and improved the functions of the immune system in fighting cancer. However, only a few patients get clinically significant benefits, ranging from an improved quality of life to lasting clinical responses, including complete remissions that can last for many months even beyond the interruption of immunotherapy. Here, we describe the clinical course of a patient with metastatic lung cancer, who had an exceptional response to combination therapy immunotherapy-chemotherapy.
\end{abstract}

Keywords: lung cancer, immunotherapy, chemotherapy, immune checkpoint inhibitors, PD-L1

\section{INTRODUCTION}

Lung cancer is the leading cause of cancer-related death worldwide [1].

Lung cancer is the leading cause of cancer death in men in Romania and more recently is a common cause of mortality among women. The main risk factor is smoking.

The prognosis of patients diagnosed with advanced lung cancer is generally considered poor, with a median survival of 8-10 months and a 2-year survival of no more than $10 \%-20 \%$ [2].

Brain metastases are a common complication in a wide range of cancers, but they are particularly common among patients with lung cancer. Approximately 10 percent of newly diagnosed patients with advanced non-small cell lung cancer (NSCLC) have brain metastases [3].
Though there are well-established management approaches for brain metastases, with local therapies as the cornerstone, treatment options for patients with advanced NSCLC and brain metastases are evolving for several reasons. First, many patients with advanced NSCLC are living several years, allowing more time for brain metastases to develop as well as for adverse effects of prior therapies for brain metastases to emerge.

The use of checkpoint immunity inhibitors in many malignancies, including lung cancer, has revolutionized the field of oncology and improved the functions of the immune system in fighting cancer. However, only a few patients get clinically significant benefits, ranging from an improved quality of life to lasting clinical responses, including complete remissions that can last for many months even beyond the interruption of immunotherapy. 


\section{CASE REPORT}

A 69-year-old woman was admitted to our hospital with cough, dyspnea, weight loss (6 kg in 2 months). A tumoral mass in the right lobe $40 / 30 \mathrm{~cm}$ with adenopatic mass, 60/45 mm in Barrety region and another adenopatic mass $80 / 45 \mathrm{~mm}$ located around the carinamass was found in the right upper lobe on computed tomography (CT), and transbronchial lung biopsy showed primary lung adenocarcinoma without epidermal growth factor receptor (EGFR) mutation and anaplastic lymphoma kinase (ALK) translocation, and the tumor was positive for programmed cell death ligand-1 (PD-L1) expression [tumor proportion score $5 \%]$. The patients is without history of smoking.

Staging was completed with a brain MRI that showed multiple metastatic lesions located frontal and in the left cerebral hemisphere and PET/CT showed spread of the primary disease in right lung, both adrenal glands, skeletal system, supradiafragmatic liver,

The final staging being T2bN3M1c, stage IVB (OSS, BRA, PUL, ADR) stage IVB.

The patient underwent whole-brain radiation therapy (WBRT) for the brain metastasis ( $10 \mathrm{~Gy} \times 3 \mathrm{fr}$, total $30 \mathrm{~Gy}$ ). We decided to start combination of Pembrolizumab with Pemetrexed and Cisplatin Q3W for $4 \mathrm{cy}$ cles and Xgeva for bone metastases. The treatment was very well tolerated without adverse reactions.

After 4 cycles of Pembrolizumab with Pemetrexed and Cisplatin we obtained a partial response to treatment with a reductions of $1 / 2$ of the target lesions, and clinical benefit (Figure1, 2).

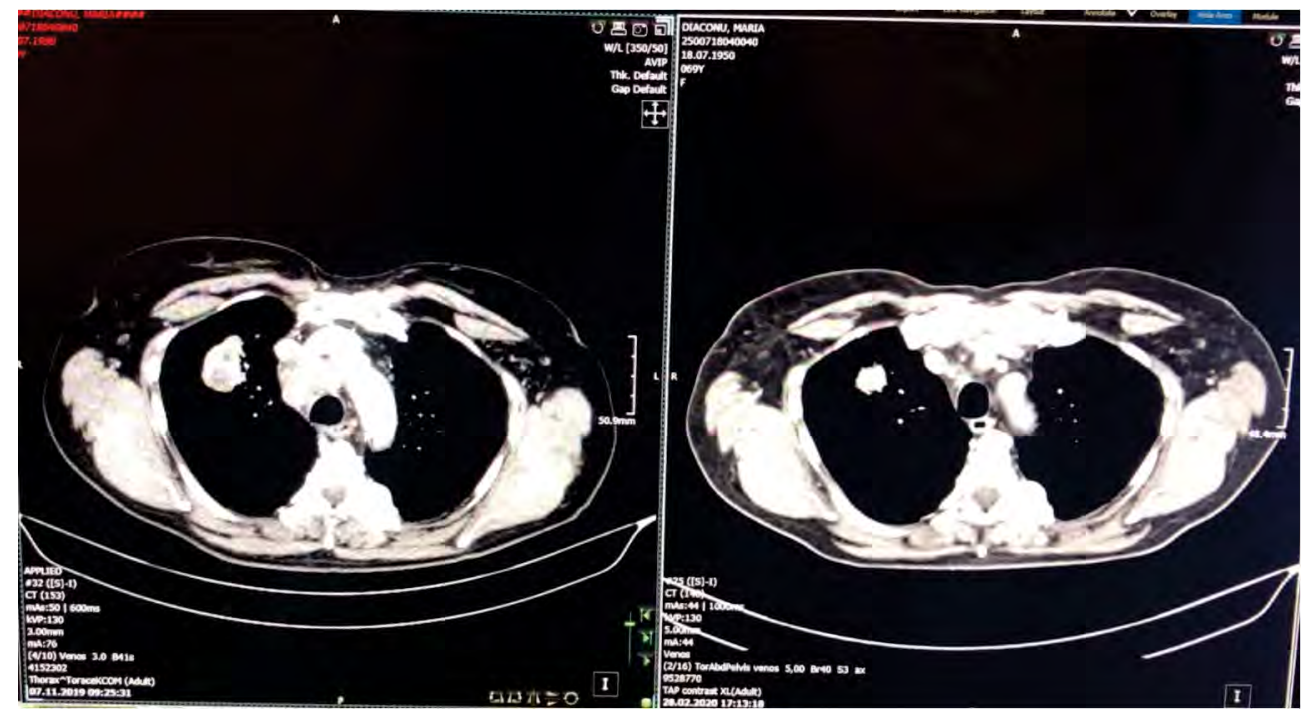

FIGURE 1. Chest computed tomography (CT) Imaging findings during the patient's course (A) at baseline (before chemotherapy) and (B) after chemoradiotherapy + immunotherapy

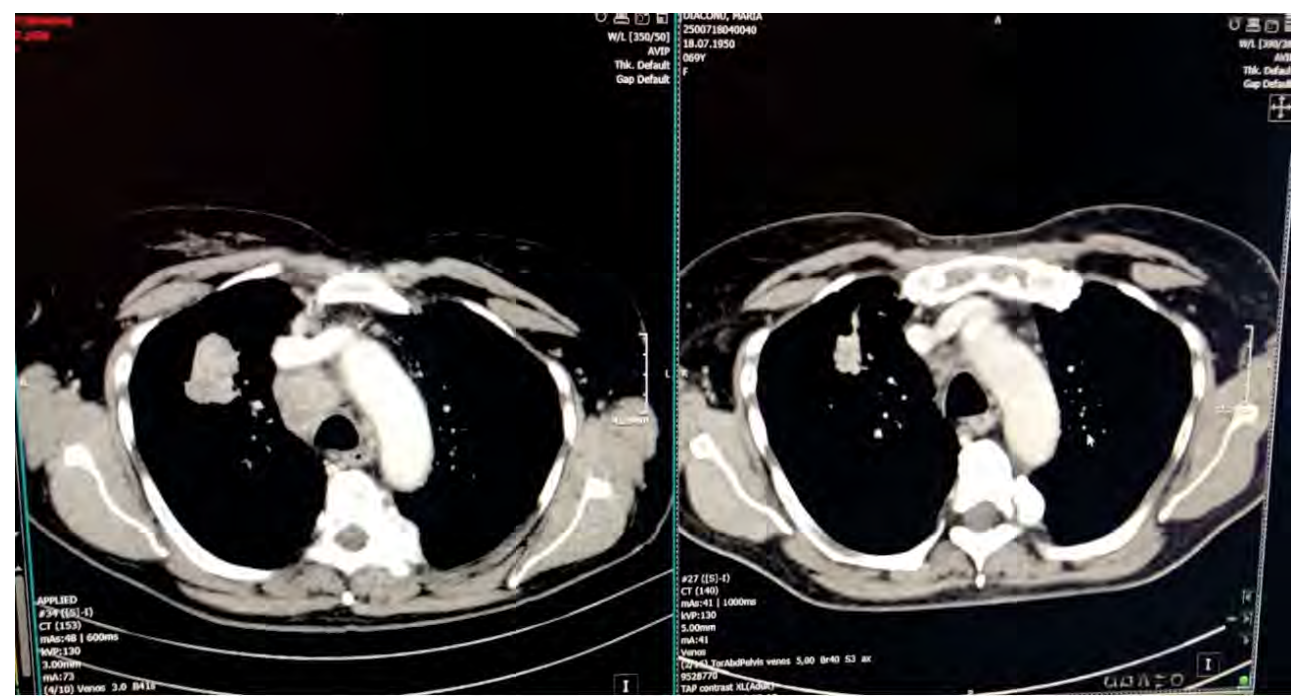

FIGURE 2. Chest computed tomography (CT) Imaging findings during the patient's course - (A) at baseline (before chemotherapy) and (B) after chemoradiotherapy + immunotherapy 

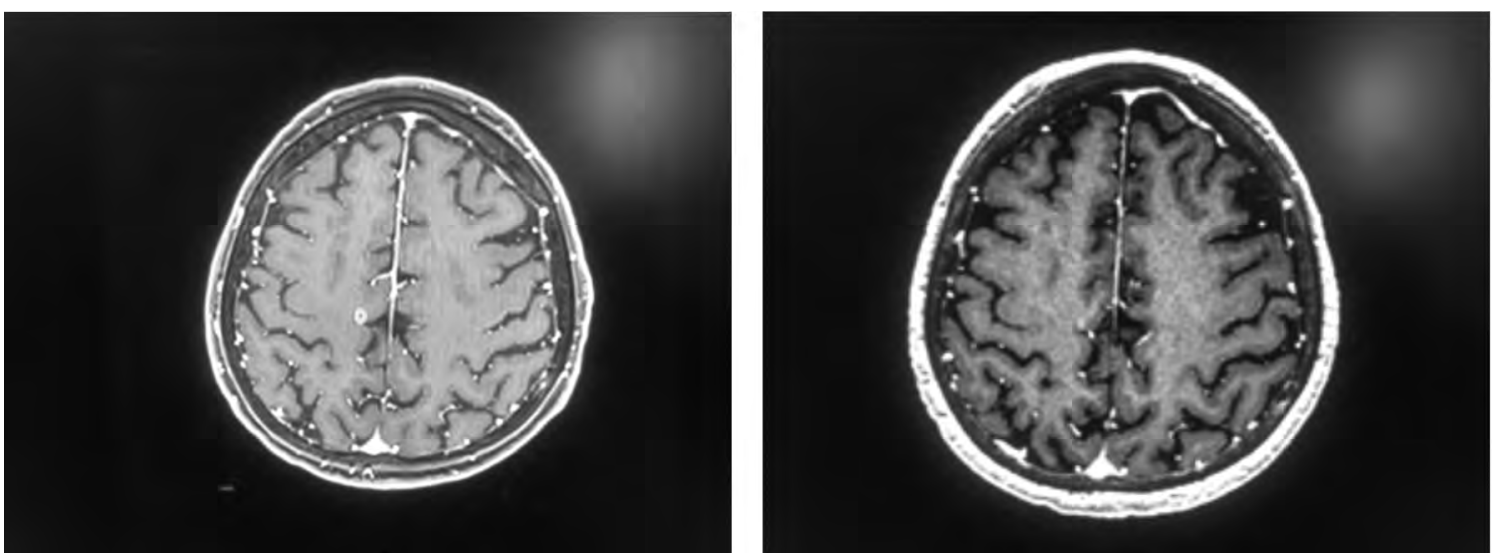

FIGURE 3. Magnetic resonance imaging scans before $(A)$ and after $(B)$ whole-brain radiation therapy (WBRT)

The CT scan showed right tumor formation at the level of LSD, anterior segment, with aspect in dimensional regression, measures $23 / 20 \mathrm{~mm}$, compared to $39 / 25 \mathrm{~mm}$. Supraclavicular, mediatinal and right paratracheal lymphadenopathy, the largest 18/16 mm compared to $65 / 45 \mathrm{~mm}$. Slightly hypetrophic adrenal glands with an unmodified dimensions compared to the previous examination. At the level of the liver, the 2 formations described above have a stationary appearance.

In the bone metastases we observed bone lesions remodeled with the increase of the osteosclerotic component, terapeutic response.

After 5 weeks, after WBRT, the multiple brain metastases show a partial response, together with the response in the lung lesions (Figure 3).

Chest and abdomen CT are being performed after another 3 months of treatment. At the time of sub-mission of this report the patient remains completely asymptomatic.

\section{DISCUSSION}

Lung cancer is the leading cause of cancer-related death worldwide [1]. The prognosis of NSCLC still remains disappointing, with a 5-year survival rate was $19 \%$ [2-3].The major benefit in the field of immunotherapy in solid tumors, including lung cancer, has been the achievement of durable responses in a subset of patients receiving checkpoint inhibitors, with subsequent translation to longer survivals. Most recently, the 5-year overall survival rate for patients treated with pembrolizumab was recently reported as $15.6 \%$ in second line of treatment and $23.2 \%$ first line in the Keynote 001 study [8]. The response to immu- notherapy depends on many factors, most notably is PDL-1 expression, male gender, smoking status, tumor mutation burden, microsatellite instability, presence or absence of $\mathrm{CD} 8+$, tumor-infiltrating lymphocytes (TILs) , EGFR status or previous radiotherapy on the tumor [10-18].

The prognosis and survival of patients with brain metastases remains poor [19]. Therapeutic options for patients with brain metastases are largely palliative and include surgical resection, whole-brain radiation therapy (WBRT), stereotactic radiosurgery (SRS) [4]. However, in the last years, many scientific efforts were in brain metastases. Multiple studies have shown Pembrolizumab has activity in brain metastases from NSCLC with PD-L1 expression at at least 1\% [20].

We wanted to increase of responses in NSCLC patients with brain metastases by using combination therapy such as immunotherapy- chemotherapy and radiotherapy and maintaining the quality of life, especially in the patients because may present neurological symptoms which can change their quality of life.

\section{CONCLUSIONS}

In conclusion, chemotherapy combined with PD-1 is a potential treatment for patients with NSCLC with multiple metastasis. After treatment with pembrolizumab and chemotherapy the patient have had long overall survival rate and PFS with high quality of life. Brain metastasis is one of the dreadful complications and a major cause of NSCLC morbidity and mortality. The prognosis after brain metastasis is extremely poor. Currently, the treatment modalities for NSCLC are limited to combined surgical resection and radiotherapy, chemotherapy and immunotherapy. 


\section{REFERENCES}

1. Bray F, Jemal A, Torre LA, Forman D et al. Long-term realism and cost-effectiveness: Primary prevention incombatting cancer and associated inequalities worldwide. J Natl Cancer Inst. 2015;107(12):djv273.

2. Ferlay J, Soerjomataram I, Dikshit R et al. Cancer incidence and mortality worldwide: Sources, methods and major patterns in GLOBOCAN 2018. Int J Cancer. 2015;136:E359-86.

3. Ettinger DS, Akerley W, Borghaei $\mathrm{H}$ et al. Non-small celllung cancer, version 2.2013 . J Natl Compr Canc Netw. 2013;11:645-53.

4. Yuankai SHI et al. China experts consensus on thediagnosis and treatment of brain metastases of lung cancer (2017 version) (in Chinese). Zhongguo Fei Ai Za Zhi. 2017;20:1-13.

5. Reck M, Rodriguez-Abreu D, Robinson AG, Hui R, Csoszi T, Brahmer JR. Updated analysis of KEYNOTE-024: Pembrolizumab versus platinum-based chemotherapy for advanced non-small-cell lung cancer with PD-L1 tumor proportion score of $50 \%$ or greater. J Clin Oncol. 2019;37:537-546.

6. Reck M, Rodriguez-Abreu D, Robinson AG, Hui R, Csoszi T, Brahmer JR.

Pembrolizumab versus chemotherapy for PD-L1-positive non-small-cell lung cancer. N Engl J Med. 2016;375:1823-1833.

7. Gandhi L, Rodriguez-Abreu D, Gadgeel S, Esteban E, Felip E, Garassino MC. Pembrolizumab plus chemotherapy in metastatic non-small-cell lung cancer. $N$ Engl J Med. 2018;378:2078-2092.

8. Pollack MH, Betof A, Dearden H, Rapazzo K, Valentine I, Shoushtari AN. Safety of resuming anti-PD-1 in patients with immune-related adverse events (irAEs) during combined anti-CTLA-4 and anti-PD1 in metastatic melanoma. Ann Oncol. 2018;29:250-255.

9. Niki M, Nakaya A, Kurata T, Yoshioka H, Kaneda T, Nomura S. Immune checkpoint inhibitor re-challenge in patients with advanced non-small cell lung cancer. Oncotarget. 2018;9:32298-32304.

10. Nomura M, Otsuka A, Kondo T, Nagai $H$, Nonomura Y, Muto M. Efficacy and safety of retreatment with nivolumab in metastatic melanoma patients previously treated with nivolumab. Cancer Chemother Pharmacol. 2017;80:999-1004.

11. Twyman-Saint Victor C, Rech AJ, Maity A, Rengan R, Pauken KE, Minn AJ. Radiation and dual checkpoint blockade activate non-redundant immune mechanisms in cancer. Nature. 2015;520:373-377.

12. Shaverdian N, Lisberg AE, Bornazyan K, Veruttipong D, Goldman JW, Lee P. Previous radiotherapy and the clinical activity and toxicity of pembrolizumab in the treatment of non-small-cell lung cancer: A secondary analysis of the KEYNOTE-001 phase 1 trial. Lancet Oncol. 2017;18:895903.

13. Borghaei $H$, Paz-Ares L, Horn L et al. Nivolumab versus docetaxel in advanced nonsquamous non-small-cell lung cancer. The New England Journal of Medicine 2015;373(17):1627-1639.

14. Rizvi NA, Hellmann MD, Snyder A et al. Cancer immunology. Mutational landscape determines sensitivity to PD-1 blockade in non-small cell lung cancer. Science 2015; 348(6230):124-128.

15. Le DT, Uram JN, Wang $\mathrm{H}$ et al. PD-1 blockade in tumors with mismatch-repair deficiency. The New England Journal of Medicine 2015; 372(26):2509-2520.

16. Tumeh PC, Harview CL, Yearley JH et al. PD-1 blockade induces responses by inhibiting adaptive immune resistance. Nature. 2014;515(7528):568-571.

17. Huang $Q$, Zhang $H$, Hai J et al. Impact of PD-L1 expression, driver mutations and clinical characteristics on survival after anti-PD-1/PD-L1 immunotherapy versus chemotherapy in non-small-cell lung cancer: A meta-analysis of randomized trials. Oncoimmunology 2018;7(12):e1396403.

18. Shaverdian N, Lisberg AE, Bornazyan K et al. Previous radiotherapy and the clinical activity and toxicity of pembrolizumab in the treatment of non-small-cell lung cancer: A secondary analysis of the KEYNOTE-001 phase 1 trial. The Lancet Oncology. 2017;18(7):895-903.

19. Giannicola R, D’Arrigo G, Pastina P et al. Correlation of survival of NSCLC patients given nivolumab with treatment relatedeosinophilia, autoimmunity and previous bevacizumab containing regimens. Journal of Clinical Oncology. 2018;36(15):e21008.

20. Goldberg SB, Schalper KA, Gettinger SN, Mahajan A, Herbst RS et al. Pembrolizumab for Management of Patients With NSCLC and Brain Metastases: Long-Term Results and Biomarker Analysis From a NonRandomised, Open-Label, Phase 2 Trial. Lancet Oncol. 2020. 